\title{
ON ITERATED SIMILARITIES OF OPERATORS ${ }^{1}$
}

\author{
DOMINGO A. HERRERO
}

\begin{abstract}
Let $\mathcal{E}(\mathcal{H})$ be the algebra of operators on a complex Hilbert space $\mathcal{H}$. If $A \in \mathcal{L}(\mathcal{X})$ is invertible and $\left\{X \in \mathcal{L}(\mathcal{X}):\left\|A^{k} X A^{-k}\right\|<C(X)<\infty\right.$, $k=0,1,2, \ldots\}$ coincides with $\mathcal{E}(\mathcal{C})$, then $A$ is a multiple of a similarity of a unitary operator.
\end{abstract}

Let $\mathcal{L}(\mathcal{H})$ be the algebra of all (bounded linear) operators acting on the complex infinite dimensional Hilbert space $\mathcal{H}$ and let $A$ be an invertible operator. In [1], J. A. Deddens analyzed the algebra $\mathscr{B}(A)=\{X \in \mathcal{L}(\mathcal{H})$ : $\left\|A^{k} X A^{-k}\right\|<C(X)<\infty$ for $\left.k=0,1,2, \ldots\right\}$ in connection with nest algebras, observed that if $A$ is a multiple of a similarity of a unitary operator, then $\mathscr{B}(A)=\mathscr{L}(\mathcal{H})$ and asked whether the converse is true. This is, indeed, the case:

Assume that $\mathscr{B}(A)=\mathscr{L}(\mathcal{H})$ and let $c \in \sigma(A)$ (the spectrum of $A$ ). Then, by the uniform boundedness principle, there exists a constant $C>0$ such that $\left\|\tau_{k}\right\|<C$ for all $k=0,1,2, \ldots$, where $\tau_{k} \in \operatorname{Aut}[\mathcal{L}(\mathcal{H})]$ is the automorphism defined by $\tau_{k}(X)=A^{k} X A^{-k}$. Thus if $\mathscr{Q}$ is the von Neumann algebra $\mathcal{L}(\mathcal{H C}) \oplus \mathfrak{L}(\mathcal{H}) \oplus \ldots$ (denumerably many copies of $\mathcal{L}(\mathcal{H})$ ), then $\tau\left(X_{0}, X_{1}, X_{2}, \ldots, X_{k}, \ldots\right)=\left(X_{0}, A X_{1} A^{-1}, A^{2} X_{2} A^{-2}, \ldots, A^{k} X_{k} A^{-k}, \ldots\right)$ $=\left(\tau_{0}\left(X_{0}\right), \tau_{1}\left(X_{1}\right), \ldots, \tau_{k}\left(X_{k}\right), \ldots\right)$ defines an automorphism of $\mathscr{Q}$ that fixes every summand of $\mathscr{Q}$ (i.e., $\tau(0,0, \ldots, 0, X$ ( $k$ th coordinate), $0, \ldots)=$ $\left(0,0, \ldots, 0, \tau_{k}(X), 0, \ldots\right)$ for all $\left.k=0,1,2, \ldots\right)$. Combining this observation with [2, Corollary 4.1.22], we see that $\tau$ is inner, i.e., there exists $T=\left(T_{0}, T_{1}, T_{2}, \ldots, T_{k}, \ldots\right)$ invertible in $\mathbb{Q}$ such that if $X=$ $\left(X_{0}, X_{1}, X_{2}, \ldots, X_{k}, \ldots\right) \in \mathbb{Q}$, then

$$
\tau(X)=T X T^{-1}=\left(T_{0} X_{0} T_{0}^{-1}, T_{1} X_{1} T_{1}^{-1}, T_{2} X_{2} T_{2}^{-1}, \ldots, T_{k} X_{k} T_{k}^{-1}, \ldots\right) .
$$

Comparing this expression of $\tau$ with its definition, it readily follows that $T_{k}=c_{k} A^{k}$ for suitably chosen constants $c_{k}, k=0,1,2, \ldots$ Since $T$ is invertible, $\sigma(T) \subset\left\{z: a=\left\|T^{-1}\right\|^{-1} \leqslant|z| \leqslant\|T\|=b\right\}$, whence we conclude that $a \leqslant\left|c_{k} c^{k}\right| \leqslant b$, because $\sigma(T) \supset \cup_{k=0}^{\infty} \sigma\left(T_{k}\right)=\cup_{k=0}^{\infty} c_{k} \sigma\left(A^{k}\right)$.

Hence, $\left\|[(1 / c) A]^{k}\right\| \leqslant b / a$ and $\left\|[(1 / c) A]^{-k}\right\| \leqslant b / a$ for all $k=$ $0,1,2, \ldots$ By a well-known theorem of B. Sz.-Nagy [3], it follows that $A=c W U W^{-1}$, where $W$ is invertible and $U$ is unitary.

The author wishes to thank the referee for the following observation: "ad hoc" modifications of the same proof yield a stronger result. Namely,

Received by the editors November 23, 1977 and, in revised form, January 24, 1978.

AMS (MOS) subject classifications (1970). Primary 47A99.

${ }^{1}$ This work has been partially supported by CONICIT (Venezuela), Grant \# 51.26.S1-0894. 
THEOREM. If $\mathscr{B}(A)$ is a $C^{*}$-algebra and every automorphism of $\mathscr{B}(A)$ has the form $\tau(X)=T X T^{-1}(X \in \mathscr{B}(A))$ for some invertible $T \in \mathcal{L}(\mathcal{H})$, then $A$ is a multiple of a similarity of a unitary operator and $\mathscr{B}(A)=\mathcal{L}(\mathcal{H})$.

The referee also remarked that J. Stampfli has independently proved that $A$ is a multiple of a similarity of a unitary operator whenever $\mathscr{B}(A)$ contains the compact operators.

\section{REFERENCES}

1. J. A. Deddens, Another characterization of nest algebras (preprint).

2. S. Sakai, $C^{*}$-algebras and $W^{*}$-algebras, Ergebnisse der Math. und ihrer Grenzgebiete, Bd. 60, Springer-Verlag, New York and Berlin, 1971.

3. B. Sz.-Nagy, On uniformly bounded linear transformations in Hilbert space, Acta Sci. Math. (Szeged) 11 (1947), 152-157.

Departamento de Matematicas, Instituto Venezolano de Investigaciones Cientificas, AP 1827, Caracas 101, Venezuela 